\title{
Algorithm of Marriage in Honey Bees Optimization Based on the Nelder-Mead Method
}

\author{
Chenguang Yang ${ }^{1,2}$ Jie Chen ${ }^{1,2}$ Xuyan $\mathrm{Tu}^{1,2}$ \\ ${ }^{1}$ Department of Automation, School of Information Science and Technology, Beijing Institute of Technology, \\ Beijing 100081, P. R. China \\ ${ }^{2}$ Key Laboratory of Complex System Intelligent Control and Decision (Beijing Institute of Technology), Ministry of \\ Education, Beijing 100081, P. R. China
}

\begin{abstract}
Marriage in Honey Bees Optimization (MBO) is a new swarm-intelligence method, but it has the shortcomings of low speed and complex computation process. By changing the structure of $\mathrm{MBO}$ and utilizing the Nelder-Mead method to perform the local characteristic, we propose a new optimization algorithm. The global convergence characteristic of the proposed algorithm is proved by using the Markov Chain theory. And then some simulations are done on Traveling Salesman Problem (TSP) and several public evaluation functions. Comparing the proposed algorithm with $\mathrm{MBO}$ and Genetic Algorithm, simulation results show that the proposed algorithm has better convergence performance.
\end{abstract}

Keywords: Marriage in honey bees optimization (MBO), Nelder-Mead Method, Markov chain, NelderMead - Marriage in Honey Bees Optimization (NMMBO), Traveling Salesman Problem (TSP)

\section{Introduction}

Swarm intelligence is a new research area. It studies the behavior of social insects and uses their models to solve problems. Recently, Based on the marriage process of honey bees, the new technique of Marriage in Honey Bees Optimization (MBO) was proposed by Jason Teo and Hussein A. Abbass[1]-[2] and has been updated by Jason Teo, Hussein A. Abbass [3] and Omid Bozorg Haddad et al [4]-[5].

The objective of this paper is to increase the performance of MBO. By combining Nelder-Mead method, an improved algorithm is proposed and its convergence is analyzed based on the theory of Markov Chain.

The paper is organized as follows. As the basis of the study, Marriage in Honey Bees Optimization (MBO) algorithm and Markov Chain with some basic theorems and definitions are reviewed respectively in
Section 2, Section 3. In Section 4 and Section 5, the proposed algorithm and its convergence analysis are presented. Finally, some simulations are done and conclusion is given.

\section{Algorithm of marriage in honey bees optimization}

The behavior of honey-bees shows many features like cooperation and communication, so honey-bees have aroused great interests in modeling intelligent behavior these years.

Marriage in Honey Bees Optimization (MBO) is a kind of swarm-intelligence method. And such swarmintelligence has some successful applications. Ant colony is an example and the search algorithm is inspired by its behavior. Mating behavior of honeybees is also considered as a typical swarm-based optimization approach. The behavior of Honey-bees is related to the product of their genetic potentiality, ecological and physiological environments, the social conditions of the colony, and various prior and ongoing interactions among these three [1]-[2].

The five main processes of $\mathrm{MBO}$ are: (a) the mating-flight of the queen bees with drones encounter at some probabilistically. (b) creating new broods $s_{-}$ the queen bees, (c) improving the broods' fitness by workers, (d) updating the workers' fitness, and (e) replacing the least fittest queen(s) with the fittest brood(s).

\section{Markov chain}

Markov chain has been widely applied to GA. Markov chains (MCs) have been used extensively to study convergence characteristic. Such as many GA methods' performance were analyzed by modeling the GA process as a Markov process.

A Markov chain is a sequence of random values whose probability at a time interval depends upon the value of the number at the previous time. The 
probabilities of a Markov chain are usually entered into a transition matrix indicating which state or symbol follows which other state or symbol.

Definition $1^{[6]}$ : A square matrix is $A=\left[a_{i j}\right]_{n \times n}$

(a) if $\forall i, j \in\{1, \ldots n\}: a_{i j}>0, \quad$ Ais positive $(A>0)$;

(b) if $\forall i, j \in\{1, \ldots n\}: a_{i j} \geq 0$, Ais nonnegative $(A \geq 0)$;

(c) if $A \geq 0$ and $\exists m \in \mathrm{N}: A^{m}>0$, Ais primitive;

(d) if $A \geq 0$ and $\forall i \in\{1, \ldots n\}: \sum_{j=1}^{n} a_{i j}=1$, Ais stochastic.

Definition $2^{[6]}$ : If the state space $S$ is finite $(|S|=n)$, and the transition probability $p_{i j}(t)$ are independent from $t$,

$$
\exists i, j \in S, \exists u, v \in \mathrm{N}, p_{i j}(u)=p_{i j}(v)
$$

the Markov chain is said to be finite and homogeneous. $p_{i j}(t)$ is the probability of transitioning from state $i \in S$ to state $j \in S$ at step $t$.

Theorem $1^{[6]}$ : For a homogeneous finite Markov chain, with the transition matrix $P=\left(p_{i j}\right)$, If

$$
\exists m \in \mathrm{N}: P^{m}>0
$$

then this Markov chain is ergodic and with finite distribution. $\lim _{t \rightarrow \infty} p_{i j}(t)=\overline{p_{j}}, i, j \in S$ is the steady distribution of the homogeneous finite Markov Chain.

Theorem $2^{[6]}$ (The basic limit theorem of Markov chain): If $P$ is a primitive homogeneous Markov chain's transition matrix, then

(a) $\exists ! \omega^{T}>0: \omega^{T} P=\omega^{T}$, $\omega$ a probability vector.

(b) $\forall \varphi_{i} \in S$ ( $\varphi_{i}$ is the start state and it's probability vector is $g_{i}^{T}$ ):

$$
\lim _{k \rightarrow \infty} g_{i}^{k} P^{k}=\omega^{T}
$$

(c) From $\lim _{k \rightarrow \infty} P^{k}=\bar{P}$, we can get a limit probability matrix $\bar{P}$,

it is a $n \times n$ matrix and it's all rows are same to $\omega^{T}$.

Theorem $3^{[6]}$ : Let $P$ be a reducible stochastic matrix, where $C: m \times m$ is a primitive stochastic matrix and $R \neq 0, T \neq 0$. Then

$$
P^{\infty}=\lim _{k \rightarrow \infty} P^{k}=\lim _{k \rightarrow \infty}\left(\begin{array}{cc}
C^{k} & 0 \\
\sum_{i=0}^{k-1} T^{i} R C^{k-i} & T^{k}
\end{array}\right)=\left(\begin{array}{cc}
C^{\infty} & 0 \\
R^{\infty} & 0
\end{array}\right)(5)
$$

is a stable stochastic matrix.

\section{Nelder-Mead method- marriage in honey bees optimization (NM- MBO)}

One of the most important advantages of MBO over Genetic Algorithm is MBO does a local search in each iteration. So $\mathrm{MBO}$ can avoid solely using crossover operator and mutation operator who is of worse local search ability.

But MBO algorithm chooses some simple and random local searching methods, such as random walk and random flip [1], which will reduce the probability of obtaining optimal solution. So such low efficiency of Worker in MBO badly influences the whole performance of $\mathrm{MBO}$.

The Nelder-Mead method is a commonly used nonlinear optimization algorithm, proposed by Nelder \& Mead.[7] It is a direct search method and does not use numerical or analytic gradients. The merit of Nelder-Mead method is that it is not sensitive to starting values and neither does it rely on derivatives nor on continuity of the objective function.

So we utilize the local search ability and replace the Worker of MBO algorithm by the Nelder-Mead method.

Some studies related to MBO have been carried out in our research. One of them is to increase the convergence speed. Here we make some introduce about it, because the main work in this paper will based on such improved MBO algorithm.

In MBO algorithm, the probability of a drone makes with a queen is defined by the annealing function [1]. Not only the calculation of probability is complex, but also its calculation participants are complicated. So the whole process has a large computation burden.

On the other hand, we have found that MBO with low speed need enough iteration times to approach optimization result. But several variables in $\mathrm{MBO}$, such as energy, speed, can't make sure about this. As the process going, the mating probability becomes smaller, which neither help the calculation process put up, nor help converge globally. So based on the original MBO algorithm, we have done some improvement on the original MBO algorithm. That is, by random initializing drones and restricting the condition of iteration, the computation process will become easier. The detail about this improvement has been discussed in other papers before.

Here we further our research to improve the performance of $\mathrm{MBO}$ and propose an algorithm of Nelder-Mead -Marriage in Honey Bees Optimization (NMMBO) by taking the Nelder-Mead method as the Worker.

The detail of NM-MBO is shown below.

Define Q: the number of queens
D: the number of drone
M: the sperm theca size
Initialize each worker with a unique heuristic
Initialize each queen's genotype at random
Apply Nelder-Mead method to improve the queen's genotype
While the stopping criteria is not satisfied (Cycle Times bigger than
Max Cycle Number or result is good enough)




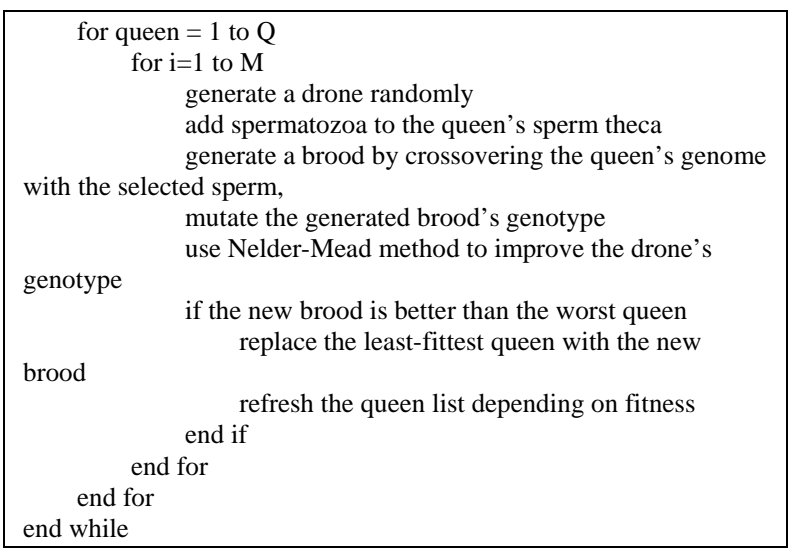

Fig. 1: Nelder-Mead- Marriage in Honey Bees Optimization algorithm (NM-MBO)

In Fig. 1, the algorithm is much easier than that in the MBO algorithm. And the number of the parameters is also less than the later. The whole process of NM-MBO has fewer complex probability calculations which will help increase the computation speed.

In NM-MBO, we define three operators: Crossover, Mutation and Heuristic. Crossover and Mutate are same as that in GA. But the Heuristic operator is a new one proposed in NM-MBO.

- Crossover: Crossover operator exchanges the pieces of genes between chromosomes. Through crossover, it introduces new chromosomes to the population, and hence the possibility of having fitter chromosomes.

- Mutation: Mutation operation alters individual alleles at random locations of random chromosomes at a very probability. It might create a better or worse chromosome, which will either thrive or diminish through next selection.

- Heuristic: Heuristic operator improves a set of broods. It help conduct local search on broods. For the good local convergence performance, we use Nelder-Mead method as the heuristic operator.

\section{Convergence analysis of NM- MBO algorithm}

In this section, we use Markov Chain to analysis the convergence of the Nelder-Mead-Marriage in Honey Bees Optimization algorithm.

There are only three ways to change from one generation to another, is Crossover, Mutate and Heuristic. These operators depend only on the inputs and not restricted with time. Then we can get the following theorem.
Definition 3: The state space of NM-MBO is

$$
X=\left\{x=\left[t_{1}, t_{2}, \ldots, t_{N}\right] \mid t_{i} \in\{0,1\}, i=1, \ldots, N\right\}(6)
$$

where $\left[t_{1}, t_{2}, \ldots, t_{N}\right]$ is the binary bit cluster listed in turn.

Define $f(x)$ as the fitness function based on $X$ and $y$ is the fitness. So the fitness aggregate $Y$ is

$$
Y=\{y \mid y=f(x), x \in X\}
$$

It is easy to see

$$
\forall x \in X, y>0
$$

Define $g=|Y|$, we can get a ordered aggregate

$$
\left\{y_{1}, y_{2}, \ldots, y_{g}\right\}, y_{1}>y_{2}>\ldots>y_{g}
$$

Crossover, Mutate and Heuristic operators lead to probable transition in the state space. And we use three transition matrix $C, M$ and $H$ to describe their infections respectively. Finally, we can get

$$
\operatorname{Tr}=C \cdot M \cdot H
$$

where $\operatorname{Tr}$ is the transition matrix of the Markov chain of the NM-MBO algorithm.

Theorem 4: The Markov Chain of NM-MBO is finite and homogeneous.

Proof:

The aggregate $\left\{x_{1}, x_{2}, \ldots, x_{M}\right\}$ is finite. So the Markov chain composed of $\left\{x_{1}, x_{2}, \ldots, x_{M}\right\}$ is finite. This finite space can also be said as a state space $X$.

With $\rho_{i}, \rho_{j} \in X$, the probability of transformation from the state $\rho_{i}$ to the state $\rho_{j}$ at step $t$ only depends on $\rho_{i}$ and is independent of time. So the Markov chain of the NM-MBO algorithm is homogeneous.

End.

Theorem 5: The transition matrixes of the crossover probability ( $C$ ) and Heuristic probability $(H)$ in the NM-MBO algorithm are all stochastic.

Proof:

The square matrix $C$ is $C=\left[C_{i j}\right]_{n \times n}$. Then

$$
\forall i, j \in\{1, \ldots n\}: c_{i j} \geq 0 \text { and } \forall i \in\{1, \ldots n\}: \sum_{j=1}^{n} c_{i j}=1
$$

So $C$ is stochastic.

The square matrix $H$ is $H=\left[h_{i j}\right]_{n \times n}$. Then,

$$
\forall i, j \in\{1, \ldots . n\}: h_{i j} \geq 0 \text { and } \forall i \in\{1, \ldots . n\}: \sum_{j=1}^{n} h_{i j}=1
$$

So $H$ is stochastic.

Theorem 6: The transition matrix of the NMMBO with mutation probability ( $M$ ) is stochastic and positive.

Proof: 
$M=\left[m_{i j}\right]_{n \times n}$ is a square matrix. Then

$$
\forall i, j \in\{1, \ldots . n\}: m_{i j} \geq 0 \text { and } \forall i \in\{1, \ldots n\}: \sum_{j=1}^{n} m_{i j}=1
$$

So $M$ is stochastic.

And the mutation has an influence on every position of a state vector. We can easily know $\forall x_{i}, x_{j} \in X$. Each position of $x_{i}$ can mutate to the value of $x_{j}$. So the probability of $x_{i}$ mutate to $x_{j}$ is positive.

So $M$ is positive.

End

Theorem 7: The Markov Chain of the NM-MBO ( $\operatorname{Tr}$ ) is ergodic and with finite distribution.. $\lim _{t \rightarrow \infty} t r_{i j}(t)=\overline{t r}{ }_{j}>0, i, j \in X$

Proof:

According to Theorem 5, Theorem 6 and(10), Tr is positive. And according to Theorem 1, this proposition is proved.

End

Definition 4: The fitness of one generation is the largest one of the individuals in this generation.

$$
f\left(\left\{x_{1}, x_{2} \ldots x_{K}\right\}\right)=\max _{i=1,2, \ldots, K}\left\{f\left(x_{i}\right)\right\}
$$

Define $X_{1}=\left\{x_{1}, x_{2}, \ldots, x_{K} \mid f\left(\left\{x_{1}, x_{2}, \ldots, x_{K}\right\}\right)=y_{i}, x_{1}, x_{2}, \ldots, x_{K} \in X\right\}$, $y_{i}$ are defined at(9), that is, the fitness of all the individuals in $X_{i}$ is equal to $y_{i}$.

Definition 5: For an arbitrary initial generation $\mathrm{X}(0), y_{1}$ is of the largest fitness,

$$
\lim _{t \rightarrow \infty} \operatorname{Pr}\left(f(X(t))=y_{1}\right)=1
$$

Then the algorithm is global convergence.

Theorem 8: The NM-MBO converges to the global optimum.

Proof:

We can define

$$
T X=\left\{X_{i} \mid i \in N\right\}
$$

For Definition 4 and Theorem 4, $T X$ is a Markov Chain. In the same time, we define

$$
P\left(X_{i}\right)=P\left\{i X \in X_{i}\right\}
$$

$i X$ is defined in (12).

We can see that $P\left(X_{i}\right)>0$ and $\sum_{i=1}^{n} P\left(X_{i}\right)=1$

Define $P\left(X_{i}, X_{j}\right)$ is the probability state $X_{i}$ go to $X_{j}$, we can get

$$
P\left(X_{i}, X_{j}\right)=\sum_{n i=1}^{N_{i}} \sum_{n j=1}^{N_{j}} P\left(x_{n i}, X_{n j}\right), x_{n i} \in X_{i}, x_{n j} \in X_{j}
$$

Because NM-MBO saves the best individual at every generation, so $P\left(X_{i}, X_{j}\right)=0, i<j$.

And the transition matrix of $T X$ 's Markov Chain can be write as follows:

$$
P=\left[\begin{array}{ccc}
P\left(X_{1}, X_{1}\right) & \cdots & P\left(X_{1}, X_{n}\right) \\
\vdots & \cdots & \vdots \\
P\left(X_{n}, X_{1}\right) & \cdots & P\left(X_{n}, X_{n}\right)
\end{array}\right]
$$

$$
=\left[\begin{array}{cccc}
1 & 0 & \cdots & 0 \\
P\left(X_{2}, X_{1}\right) & P\left(X_{2}, X_{2}\right) & \ddots & \vdots \\
\vdots & \vdots & \ddots & 0 \\
P\left(X_{n}, X_{1}\right) & \cdots & \cdots & P\left(X_{n}, X_{n}\right)
\end{array}\right]
$$

For Theorem 3,

$$
\begin{gathered}
C=1, T=\left[\begin{array}{ccc}
P\left(X_{2}, X_{2}\right) & \cdots & 0 \\
\vdots & \ddots & \vdots \\
P\left(X_{n}, X_{2}\right) & \cdots & P\left(X_{n}, X_{n}\right)
\end{array}\right], R=\left[\begin{array}{c}
P\left(X_{2}, X_{1}\right) \\
\vdots \\
P\left(X_{n}, X_{1}\right)
\end{array}\right] \\
P^{\infty}=\lim _{k \rightarrow \infty} P^{k}=\lim _{k \rightarrow \infty}\left(\begin{array}{cc}
C^{k} & 0 \\
\sum_{i=0}^{k-1} T^{i} R C^{k-i} & T^{k}
\end{array}\right)=\left(\begin{array}{ll}
C^{\infty} & 0 \\
R^{\infty} & 0
\end{array}\right)
\end{gathered}
$$

For Theorem 7 and Theorem $1, P^{\infty}$ is a stable random matrix, So $R^{\infty}=1$. That is

$$
R^{\infty}=\lim _{k \rightarrow \infty} R^{k}=\left[\begin{array}{c}
\lim _{k \rightarrow \infty}\left(P\left(X_{2}, X_{1}\right)\right)^{k} \\
\vdots \\
\lim _{k \rightarrow \infty}\left(P\left(X_{n}, X_{1}\right)\right)^{k}
\end{array}\right]=\left[\begin{array}{c}
1 \\
\vdots \\
1
\end{array}\right](22)
$$

So every state in $T X$ will go to $X_{1}$, if the iteration number is big enough, this proposition is proved.

End

\section{Simulation}

To test the convergence performance of NM-MBO, we choose original MBO algorithm and Genetic Algorithm for comparison.

We did the simulation on two parts, one using some popular complex Evaluation Functions and the other using Traveling Salesman Problem (TSP).

\subsection{Comparison on evaluation functions}

The initial value is generated randomly, and each figure shows the average results of 20 times simulation with one Evaluation Function.

- Evaluation Function 1: Sphere Model

$$
f(x)=\sum_{i=1} x_{i}^{2}, \quad\left|x_{i}\right| \leq 100
$$




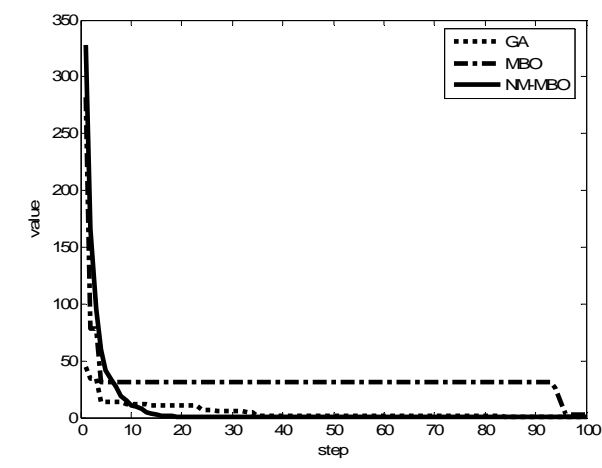

Fig. 2: Results of NM-MBO, MBO and GA with evaluation function 1.

- $\quad$ Evaluation Function 2: Schwefel’s Problem 1

$$
f(s)=\sum_{i=1}^{30}\left|x_{i}\right|+\prod_{i=1}^{30}\left|x_{i}\right|, \quad\left|x_{i}\right| \leq 10
$$

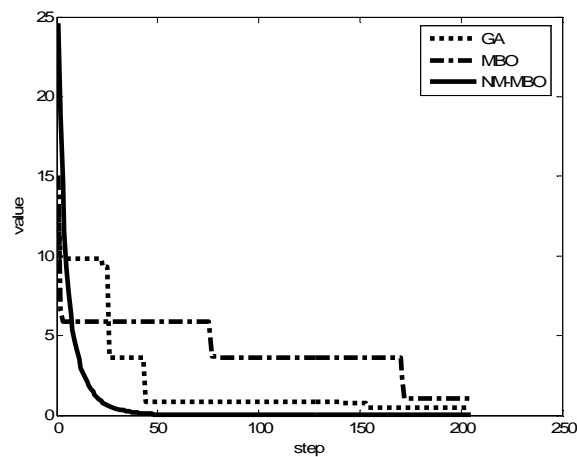

Fig. 3: Results of NM-MBO, MBO and GA with evaluation function 2 .

- $\quad$ Evaluation Function 3: Schwefel's Problem 2

$$
f(x)=\sum_{i=1}\left(\sum_{j=1}^{i} x_{j}\right)^{2}, \quad\left|x_{i}\right| \leq 100
$$

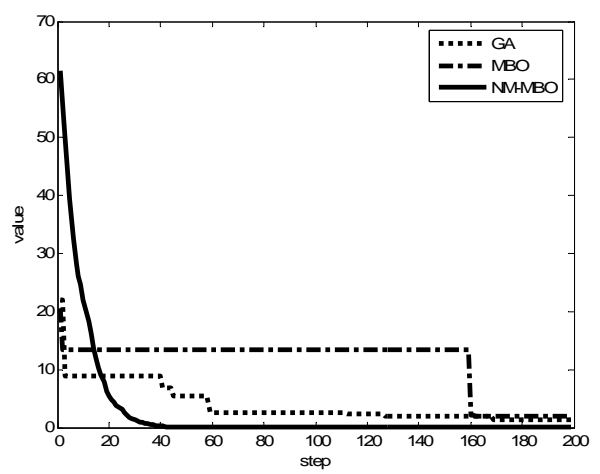

Fig. 4: Results of NM-MBO, MBO and GA with evaluation function 3 .

- $\quad$ Evaluation Function 4: Schwefel's Problem 3

$$
f(x)=\max _{i=1}\left\{\left|x_{i}\right|\right\}, \quad\left|x_{i}\right| \leq 100
$$

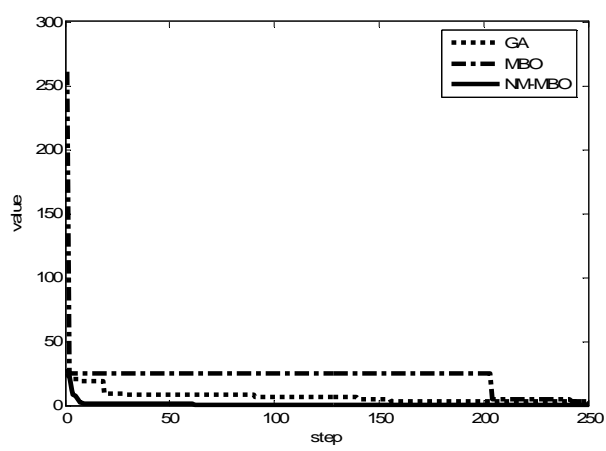

Fig. 5: Results of NM-MBO, MBO and GA with evaluation function 4 .

- Evaluation Function 5: Generalized Rosenbrock's Function

$$
f(x)=\sum_{i=1}\left[100\left(x_{i+1}-x_{i}^{2}\right)^{2}+\left(1-x_{i}\right)^{2}\right], \quad\left|x_{i}\right| \leq 30 \text { (27) }
$$

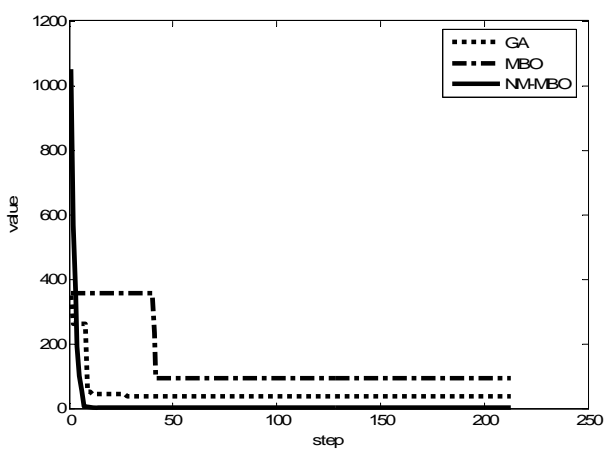

Fig. 6: Results of NM-MBO, MBO and GA with evaluation function 5 .

- $\quad$ Evaluation Function 6: Step Function

$$
f(x)=\sum_{i=1}^{30}\left(\left\lfloor x_{i}+0.5\right\rfloor\right)^{2}, \quad\left|x_{i}\right| \leq 100
$$

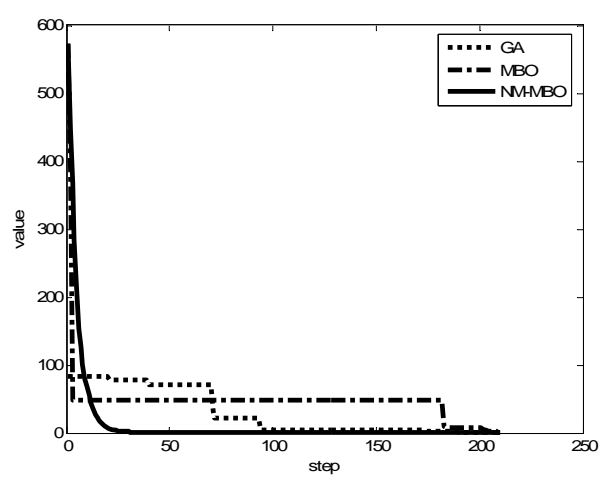

Fig. 7: Results of NM-MBO, MBO and GA with evaluation function 6 .

- Evaluation Function 7: Generalized Rastrigin’s Function 
$f(x)=\sum_{i=1}\left[x_{i}^{2}-10 \cdot \cos \left(2 \pi x_{i}\right)+10\right], \quad\left|x_{i}\right| \leq 5.12$

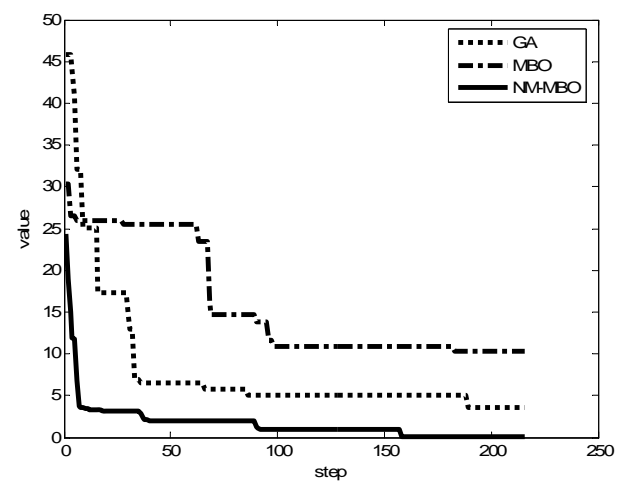

Fig. 8: Results of NM-MBO, MBO and GA with evaluation function 7 .

- Evaluation Function 8: Ackley's Function

$$
f(x)=\frac{1}{4000} \sum_{i=1} x_{i}^{2}-\prod_{i=1} \cos \left(\frac{x_{i}}{\sqrt{i}}\right)+1,\left|x_{i}\right| \leq 600
$$

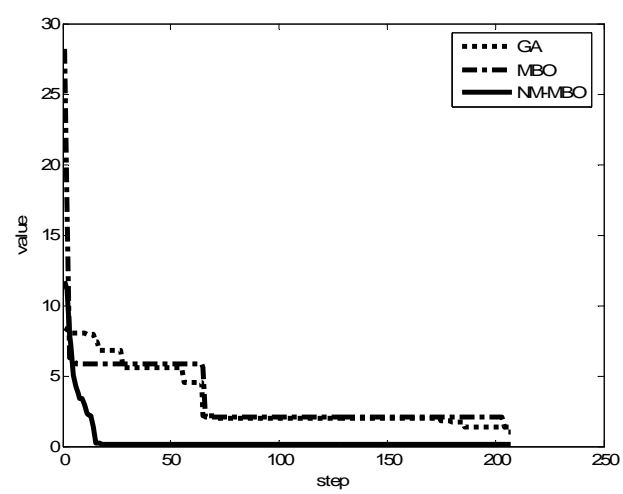

Fig. 9: Results of NM-MBO, MBO and GA with evaluation function 8.

\subsection{Traveling salesman problem}

A Classical Traveling Salesman Problem (TSP) has been an interesting problem. Given a number of nodes and their distances of each other, an optimal travel route is to be calculated so that starting from a node and visit every other node only once with the total distance covered minimized.

Here TSP based on the data form TSPLIB is solved by NM-MBO, MBO and GA algorithm respectively.

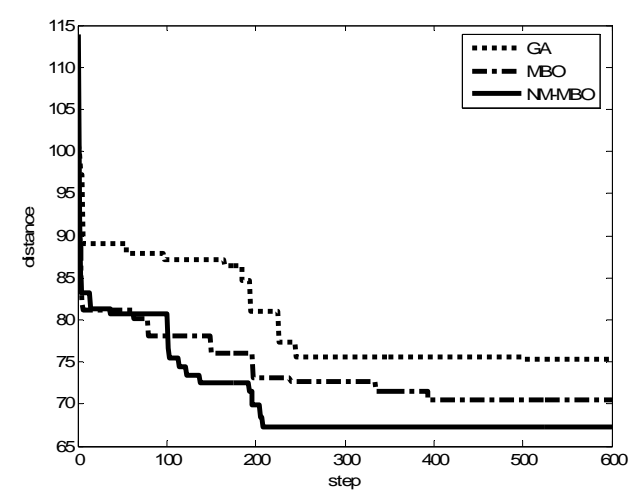

Fig. 10: TSP with 16 nodes solved by NM-MBO, MBO and GA.

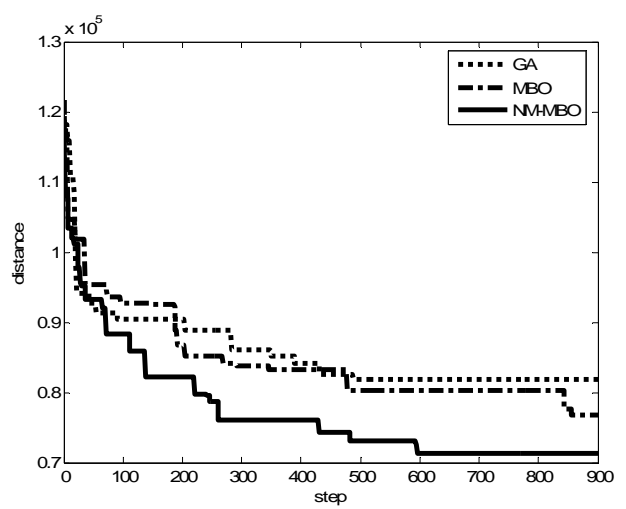

Fig. 11: TSP with 48 nodes solved by NM-MBO, MBO and GA.

\subsection{Some remarks}

From the above, we can see that the NM-MBO show better performance than $\mathrm{MBO}$ and GA, not only to solve TSP but also to optimize the evaluation function, and can keep converge faster with different node's number.

The simulation results show that:

- NM-MBO is convergent and keeps good performance for all these test functions, though these test function are more complex than the normal ones and may have many local optimization points.

- $\quad$ NM-MBO performs better than MBO and GA. NM-MBO converges more quickly, especially at initial part. Particularly, even if the initial condition is worse than MBO and GA, NMMBO can show finer result.

- As for $\mathrm{MBO}$, because of the process of choosing drones with some probability, MBO's performance is not always well. It often keeps staying at a value for some time and then drops dramatically at some step. 
Sometimes $\mathrm{MBO}$ is better than GA, but sometimes not.

\section{Conclusions}

Convergence performance is very important for optimization methods. In this paper, we proposed an algorithm of Nelder-Mead Method in Honey Bees Optimization (NM-MBO) to overcome the slowness of the original MBO.

MBO has a set of parameters to coordinate and much of calculation time is cost. While, NM-MBO avoids such complex process and also can reach the expect result. It generates a drone randomly each time and mate with a finite quantity of queens. So NMMBO can not only avoid the local optimum, but also increase the speed. Also NM-MBO is easy to implement and has few parameters to adjust. And the global convergence is preserved for optimization. Simulating with complex evaluation functions and TSP, NM-MBO shows better performance than MBO and GA.

The algorithm still deserves deep study. And the research about NM-MBO will be carried out and will be tested and improved with practical cases in the future.

\section{Acknowledgement}

This work is partially supported by Beijing Priority Laboratory Fund of China (Grant No.SYS10070522).

\section{References}

[1] H.A. Abbass, Marriage in Honey Bees Optimization (MBO): a haplometrosis polygynous swarming approach. Congress on Evolutionary Computation, CEC2001, Seoul, Korea, pp. 207-214, 2001.

[2] H.A. Abbass, A single queen single worker honey-bees approach to 3-SAT. Proceedings of the Genetic and Evolutionary Computation Conference, GECCO2001, San Francisco, USA, pp.807-814, 2001.

[3] J. Teo and H.A. Abbass, An annealing approach to the mating-flight trajectories in the marriage in honey bees optimization algorithm, Technical Report CS04/01, School of Computer Science, University of New South Wales at ADFA, 2001.

[4] O. Bozorg Haddad, A. Afshar and A.Miguel Marino, Honey-bees mating optimization (HBMO) algorithm: a new heuristic approach for water resources optimization. Water Resources Management, 20:661-680, 2006.
[5] H.S. Chang, Converging marriage in honey-bees optimization and application to stochastic dynamic programming. Journal of Global Optimization, 35: 423-441, 2006.

[6] G. Rudolph, Convergence analysis of canonical genetic algorithms. IEEE Transaction Neural Networks. 5(1): 96-101, 1994.

[7] J.C. Lagarias, J.A. Reeds, M.H. Wright and P.E. Wright, Convergence properties of the NelderMead simplex method in low dimensions, SIAM Journal of Optimization, 9(1): 112-147, 1998. 